\title{
Mecanismos de Cooperación Universidad - Empresa: Estructuras de Transferencia y Difusión Tecnológica ${ }^{1}$
}

\section{Carlos Martínez Pávez*}

\footnotetext{
Ingeniero Civil Químico de la Universidad de Concepción, Chile. Ph.D en Ingeniería Química y Procesos de la University of Strathclyde, Gran Bretaria. Decano de la Facultad de Ingeniería de la Universidad Mayor, Chile. Director del Programa de Gestión Tecnológica del Centro Interuniversitario de Desarrollo - CIMDA. Coordinador de Proyectos de la Red ALFA de la Unión Europea y de la Agencia Española de Cooperación Internacional - AECI.
}

\section{Resumen}

El actual sistema de globalización económica demanda, para los países latinoamericanos, estrechar el vínculo universidad - empresa mediante una visión competitiva expresada en: Investigación y Desarrollo - I \& $D$; innovación, difusión y transferencia tecnológica; inversión financiera y alta calidad de los recursos humanos.

Es por esto, que el presente documento hace énfasis en los mecanismos de cooperación o estructuras de

1 Anexo del Documento Cooperación universidad - empresa: contexto y perspectivas para su desarrollo en América Latina. En: SEMIMARIO INTERMACIOMAL COOPERACIOM UMIVERSIDAD - EMPRESA: COMTEXTO Y ESTRATEGIAS DE DESARROLLO. (1997:Monterrey, México). Memorias Seminario internacional cooperación universidad - empresa: contexto y estrateglas de desarrollo. México: Instituto Tecnológico y de Estudios Superiores de Monterrey, 1997 
transferencia tecnológica de la universidad a la empresa, mostrando modelos implementados en países como Italia, Holanda, Francia, Gran Bretaña y Alemania. En especial, expone los parques tecnológicos y las incubadoras de empresas como proyectos que, además de articular la oferta académica con la demanda del sector empresarial, impulsan el desarrollo de la región involucrada. Finalmente, esboza los aspectos jurídicos establecidos en los vínculos contractuales entre universidad y empresa.

\section{Abstract}

The current system of economic globalization requires for the Latin-american countries to narrow the university-enterprise tie through a competitive view expressed in: research and development; technological innovation, spreanding and transference; financial investment and human resourcees of hight quality.

Thas is why, this document makes emphasis upon the cooperation mechanisms or structures of technological transference from university to enterprise, showing patterns implemented in countries such as Italy, Metherlands, France, England and Germany. Specially, it presents the technological parks and incubators of enterprises like projects that, in addition to match the academic offer with the demand of the enterpreuner sector, promote the development of the involved region. Finally, it outlines the juridical aspects established in the current ties betwen university and enterprise.

\section{Generalidades}

En América Latina, alrededor del $65 \%$ de la capacidad científica y tecnológica está en la universidad. Sin embargo, la aplicación o explotación de los resultados de la relación Investigación y Desarrollo - I \& D realizada en ella no es efectiva, debido principalmente a mecanismos de transferencia y difusión tecnológica ineficientes, y a la desconexión entre las necesidades del sector productivo de bienes y servicios y la investigación universitaria. También, a la carencia de apropiados instrumentos financieros para desarrollar investigación tecnológica que pueda impactar las bases industriales.

En la actualidad se observa en las universidades latinoamericanas, una preocupación por incrementar sus nexos con la empresa de una forma más sistemática. Así, siguiendo los ejemplos de universidades de países desarrollados han cambiado su actitud frente al vínculo universidad - empresa, llegándolo a transformar en una cultura de vida.

La reciente ejecución de varios proyectos con alcance internacional, ha establecido la necesidad de crear conciencia sobre cómo en los escenarios futuros, la innovación tenológica, la calidad de los recursos y la difusión de las nuevas tecnologías, jugarán un papel primordial en el desarrollo; para ello se requerirá de profesionales que puedan asumir funciones bajo estas realidades, como determinar el tipo de tecnología más adecuado, su asimilación y difusión, además de adaptar, mejorar y desarrollar tecnología. En este sentido, la universidad deberá, necesariamente, adoptar como estrategia: la proyección de sus recursos humanos al sector productivo; capacidades institucionales y conocimientos; proveer servicios científicos y tecnológicos de apoyo a la exportación como infraestructura de servicios de información técnica; I \& D; control de calidad; normalización y metrología. Hoy se observa claramente que las empresas dependen fuertemente de los conocimientos generados en la universidad y centros tecnológicos, como de la calidad de sus recursos humanos.

Por lo anterior, las universidades deben adoptar formas de respuesta dirigidas a: 1) Mejorar las actividades de difusión del quehacer universitario en lo relativo a su experiencia para satisfacer necesidades de la empresa, 2) Proveer apoyo profesional y financiero para la transferencia tecnológica desde la universidad a la empresa, mediante oficinas de vinculación o enlace que articulen las demandas de las empresas con la oferta universitaria, 3) Establecer empresas conjuntas para explotar los resultados de la investigación universitaria y centros de investigación orientados a la solución de problemas tecnológicos de la empresa, 4) Cooperar con otras instituciones para compartir experiencias y capacidades.

Por otra parte, se empiezan a estudiar nuevas formas de cooperación universidad - empresa, nuevos diseños de instrumentos y mecanismos financieros para apoyar, en forma específica, las necesidades de los actores del Sistema de Innovación; al mismo tiempo, se está dando especial atención a los aspectos legales de la propiedad industrial y al mantenimiento de un clima propicio para la innovación tecnológica. 
Para consolidar la cooperación es necesario promover al interior de las universidades y en instancias de gobierno, la "idea-fuerza" de que las relaciones universidad-empresa-estado, constituyen un instrumento para la adquisición y desarrollo de ventajas competitivas, en el actual escenario internacional en la medida en que: 1) La universidad fortalezca y consolide la excelencia académica y oriente sus esfuerzos de I \& D a la solución de los problemas del sector productivo y los del propio Estado, 2) La empresa desarrolle una cultura por la innovación y competitividad, para lo cual se requiere de una mayor profesionalización y calificación de sus cuadros gerenciales y de su fuerza de trabajo, así como del fortalecimiento de sus capacidades para incorporar, adaptar y generar tecnología, 3) El Estado cree un entorno adecuado para el desarrollo científico-tecnológico estableciendo políticas, mecanismos e instrumentos que favorezcan la innovación y competitividad, el gasto en I \& D y la inversión en tecnologías competitivas. Para esto, la universidad debe ampliar su horizonte de inter-relación, saliendo del ámbito académico para atacar los problemas del medio en lo relativo a formación, servicios científico-tecnológicos y transferencia y difusión tecnológica.

Existen muchas formas exitosas de mejorar los vínculos, la transferencia y difusión de la tecnología. En algunos casos, es oportuna la I\&D cooperativo, en otros el establecimiento de una empresa conjunta para explotar comercialmente una innovación tecnológica; también frecuentemente y a bajo costo, los cursos de entrenamiento para transferir conocimiento sobre nuevos procesos tecnológicos. Mo existe un lugar único donde se pueda llevar a cabo la transferencia; puede ser tan efectivo realizarla en la universidad, en la industria, en centros de I \& D, o en general, en cualquier mecanismo de cooperación.

Como ejemplos, se pueden señalar algunas estructuras que ejecutan actividades de I \& D conjuntas, estas son las asociaciones de interés económico y científico entre universidades y empresas establecidas en Francia, y los consorcios universidad -empresa de Italia y Alemania; otras formas buscan principalmente dinamizar la acción de centros e institutos de consultoría de servicios y de formación, como aquellos que operan en Gran Bretaña, Holanda, Francia, Bélgica, Italia y Alemania. También, existen centros regionales de información y transferencia tecnológica en países como Francia y Dinamarca para apoyar al sector empresarial. Por último, en el contexto universitario y de orga- nismos públicos se han puesto en marcha unidades de vinculación en la industria, fundaciones y otras organizaciones creadas en casi toda Europa para la interrelación con la empresa.

\section{Tipos de mecanismo de cooperación}

Los mecanismos de cooperación o estructuras de transferencia, pueden ser de diferente naturaleza y tienen como misión articular, en forma eficiente y transparente, los requerimentos o demandas externas específicas con la oferta de la universidad.

En el ámbito de sus funciones, el mecanismo, en primer término, formaliza las ofertas de las capacidades de la universidad buscando la cooperación con la empresa; ello significa que debe establecer bases de datos con la oferta universitaria en cuanto a su localización, capacidad física y recursos humanos disponibles. En segundo término, impulsa y dinamiza la transferencia de los resultados de IQD de la universidad y su aplicación en la empresa. Por último, facilita la difusión, conocimiento e inter-relación de la universidad dentro del Sistema de Innovación, esta función implica actividades promocionales o de sensibilización y de contacto, para crear la imagen institucional como: lobbing, desayunos y almuerzos de trabajo con empresarios, folletos, páginas editoriales sobre temas de relevancia y actualidad.

En el contexto internacional se encuentran los siguientes mecanismos:

Oficinas de Cooperación Industrial o Enlace: Sus funciones básicas son la identificación de los recursos disponibles, la creación y mantenimiento de bases de datos y de otras formas de información requerida. Además de promover y comercializar los conocimientos científico - tecnológicos y servicios, negociar y asesorar sobrecontratos e identificar fuentes de financiamiento para llevar a cabo actividades conjuntas con la empresa.

Centros de Cooperación Industrial: Estos Centros normalmente realizan las mismas funciones de una Oficina de Enlace, con una misión adicional de intermediación industrial para ayudar a la identificación de las necesidades reales de las industrias y facilitar la cooperación o ayuda necesaria. Se requiere que el personal encargado de estas funciones, sea de la más alta capacidad y competencia técnica. 
Centros de Investigación Industrial: Estos se caracterizan por poseer capacidad de especialización e interdisciplina, para satisfacer eventuales demandas de las industrias y responder en forma oportuna a requerimientos específicos.

Asociados industriales o clubes: Un número importante de instituciones de educación superior ha seguido la estrategia de suministrar conocimientos en investigación básica y aplicada, que puedan orientarse hacia las necesidades de un determinado sector industrial o de una empresa, en particular. Esta acción se ejecuta a través de reuniones técnicas entre el grupo de investigadores y el de la industria. Las reuniones y visitas, tanto en el campus como en la empresa, permiten identificar problemas y posibles soluciones, así como transferir información y acrecentar los vínculos personales.

\section{Estructuras internas para transferencia de tecnolo-} gía: Se ocupan de la cooperación general y del proceso de transferencia mismo. Su principal función es la interacción eficiente con organizaciones externas dando como resultado la beneficiosa transferencia de derechos de propiedad intelectual y de otros servicios. Para cumplir estas funciones, el personal debe identificar, en forma temprana, la idea y su potencial de desarrollo para luego proteger el invento, asesorar sobre aspectos legales, negociar acuerdos, comercializar los resultados y conocimientos técnicos de la investigación, identificar socios industriales, obtener financiamiento para I \& D y, crear y desarrollar otras estructuras de transferencia o empresas para explotar comercialmente las innovaciones producidas.

Estructuras autónomas de transferencia tecnológica: Estas estructuras, situadas fuera de la administración institucional, pueden enfrentar en mejor forma la gestión de la transferencia a la empresa. Aunque sus funciones son idénticas a aquellas de las Estructuras Internas, su independencia les trae ventajas en la constitución de los órganos de dirección y gerencia, en sus planes de desarrollo y en el uso de los conocimientos, sin las limitaciones académicas y organizacionales de la universidad.

Centros de inventos: Estos tienen por propósito crear la necesaria base innovadora para la identificación y desarrollo de una idea, facilitar el trabajo comercialmente creativo del personal, generar espíritu empresarial y prestar servicios a los inventores, empresarios o empresas innovadoras. Además, poseen la ca- pacidad para identificar, seleccionar y evaluar las ideas novedosas, incluyendo pruebas y construcción de prototipos, proporcionar información sobre propiedad intelectual, asesoría en contratos de licencias y de financiación, administración de innovaciones, cursos y seminarios.

Centros de incubación o incubadoras de empresas: Estos centros están en condiciones de proveer varios de los servicios antes señalados para un Centro de Inventos. Sin embargo, se diferencian del anterior en las facilidades de espacio físico para el período de gestación de la empresa, hasta que la tecnología llega al mercado. Se reconoce que la mayor parte de las universidades sólo están en capacidad de emprender y enfrentar exitosamente las etapas iniciales del ciclo de innovación ( hasta la fecha de laboratorio), donde posteriormente, muchas tecnologías son abandonadas por falta de finaciación, de conocimiento del mercado o de interés de la industria. El Centro de Incubación puede suministrar un ambiente apropiado con espacio fisico a bajo costo, facilidades administrativas y acceso a asesoría científica y tecnológica, técnica, legal, financiera y otras actividades contratadas. Existe una reglamentación muy estricta en cuanto a la admisión y permanencia en el Centro.

Empresas derivadas o conjuntas: Estas empresas están formadas por inventores y empresarios, muchos de éstos provienen de la universidad y que han evolucionado desde las empresas de asesoría o consultoría (empresas blandas) de académicos, ubicadas dentro o fuera del campus, hasta empresas más riesgosas (duras) de servicio comercial o manufactura. Por 10 general, los académicos desean conservar el control de "su empresa", pero por su falta de aptitud gerencial y empresarial, podrían llegar al fracaso. La participación de la universidad en estas empresas surge a menudo, si es dueña de la propiedad intelectual del producto, a comercializar y para lo cual se requiere la licencia; las experiencias conocidas indican que esta unión mejora sustancialmente el desarrollo de las empresas, por cuanto el otro socio tiene la experiencia comercial y de mercado, de la cual carecen la universidad y sus académicos.

\section{Parques científicos, de investigación o tecnológicos:} Esta estructura es un mecanismo adoptado para mejorar el proceso de transferencia y difusión de tecnología, promover la creación de nuevas empresas y consolidar el desarrollo de las existentes. Los Parques más exitosos han sido aquellos donde los criterios de ocupa- 
ción del mismo han sido claramente identificados y respetados estrictamente y en el cual las empresas que ocuparon el Parque, han tenido intereses de I \&D vinculados con los conocimientos académicos existentes en el mismo. Estos Parques requieren de la participación de una institución de educación superior con una sólida base de investigación; de ta presencia y:participación de una masa crítica de académicose investigadores, empresarios y personal técnico, fuentes de financiamiento, sistemas de información, acceso a las instalaciones, apoyo administrativo y facilidades de acomodación junto a la calidad de vida del en-

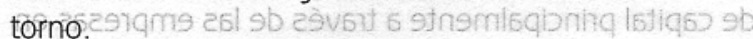
gupisa lo ne sup zetnis sipolonjat bils gb jotoge is

Unidades de entrenamiento industrial: Esta estructura de transferencia se ha dado en dos formas: la primera, como una simple interacción entre la universidad y la empresa, a través de los académicos, en proyectos orientados a mejorar el funcionamiento y la productividad industrial, así como el entrenamiento de los profesionales de la industria. También existen programas de intercambio de profesionales para beneficio mutuo de la enseñanza y la investigación. La segunda, consiste en la creación de estructuras específicas orientadas a la formación y capacitación.

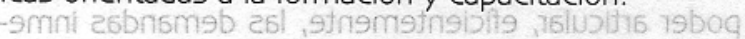



Formas modernas:

\section{Parques tecnológicos e incubadores de empresas}

En los países desarrollados, los parques tecnológicos se han constituído en un importante mecanismo de tecnología. El boom de las empresas de alta tecnología en estos países se debe en gran parte a la existencia de universidades fuertemente vinculadas y motivadas hacia la implantación de los conocimientos, por ellas generados, en empresas existentes o en nuevas empresas de bases tecnológicas; creadas por generaciones de noveles empresarios cuyas características fundamentafes son sujuventud, disposición y audacia.

En cada caso, se ha requerido de actores fuertemente motivados únos hacia la búsqueda de la aplicación de sus conocimientos, otros a satisfacer sus necesidades tecnológicas para enfrentarse en mejor forma a los mercados, algunos para independizarse y ser sus propios empleadores, muchos para emplear sus recursos financieros en inversiones muy diferentes a las tradicionales y finalmente, el Estado para crear mayores oportunidades de desarrollo, vía la creación de empresas, especialmente en zonas geográficas deprì midas.

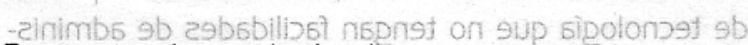
En cuanto a las tipologías de parques tecnológicos, es posible distinguir las siguientes:

roingque noì

Centro de innovaciōn : Este mecanismo relativamente pequeño, posee facilidades que permiten el desarrollo de ideas de negocios, pero no provee acomodación cuando estos negocios han crecido 0 bien para empresas existentes de tamaño medio 0 grande.

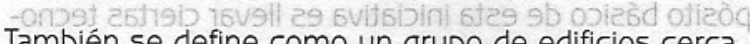
nos a un centro académico de excelencia que provee acomodación y facilidades administrativas para el seguimiento de investigación estratégica o desarrollo de prototipos. Un Centro de Innovación forma parte normalmente de las actividades regionales.

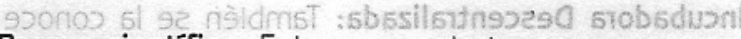
Parque científico: Este se caracteriza porque provee acomodación para los negocios emergentes y para los que existen. Mormalmente, es un sitio con atractivo (green field) donde se puede llevar a cabo la pequeña manufactura, se diferencia del Parque de Investigación, en el hecho de que este último prohibe todo tipo de manufactura y la producción de prototipos.

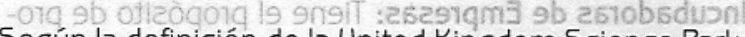
Según la definición de la United Kingdom Science Park Association, un Parque Científico es un desarrollo urbanístico que incluye características como: vínculos formales y operacionales con universidades o centros de investigación, está diseñado para promover la formación y crecimiento de negocios basados en 'conocimiento' y otras organizaciones. Además, tiene un función administrativa que está vinculada en forma activa a la transferencia de tecnología y habilidades de negocios hacia las organizaciones existentes en el Parque. Esta definición excluye aquellos mecanismos de desarrollos que se orientan sólo como proyectos para la ubicación de empresas de alta tecnología (parque industrial) sin que exista un estímulo organizacional o de facilidades de acceso a tecnología.

Parque de negocios: Es un dispositivo que provee acomodación de alta calidad en el cual puede tener lugar una gran variedad de actividades tales como las de manufacturas, exhibiciones, distribuciones, entre otras; de ahí que este Parque no requiera estar localizado cerca de una institución de educación superior o tener residentes que estén vinculados exclusivamente en industrias de alta tecnología. 
Parque tecnológico: Es esencialmente un mecanismo de desarrollo urbanístico, orientado a empresas de tecnología que no tengan facilidades de administración y estén vinculadas con instituciones de educación superior.

De igual forma, las Incubadoras de Empresas pueden adquirir varias formas:

Centro de Excelencia: Caracterizado por un fuerte apoyo privado y vínculos estrechos con universidades o programas de investigación gubernamentales. El propósito básico de esta iniciativa es llevar ciertas tecnologías a la fase de comercialización.

Centro de Innovación: Mo se orienta al desarrollo de nuevas tecnologías sino a aplicaciones más eficientes de tecnologías conocidas o de innovación.

Incubadora Descentralizada: También se la conoce como "Incubadora sin Paredes", donde los empresarios no están localizados en un sitio común, sino que están esparcidos en diferentes sitios y cuyo servicio de entrenamiento y administración, se les suministra en forma descentralizada; este es un ejemplo típico en áreas rurales.

Incubadoras de Empresas: Tiene el propósito de proveer de espacio común y una serie de servicios compartidos a los nuevos empresarios. Existe en este caso, reglamentación referente a las condiciones de entrada y salida para aquellos emprendedores que se vinculan a la incubadora, dependiendo de las características propias de la misma.

En cada una de estas variedades de incubadora, el factor común lo constituye la provisión de espacio apropiado y servicios compartidos, todos entregados a precios convenientes que permiten a los nuevos empresarios orientar todo su esfuerzo, con más tranquilidad, hacia los aspectos técnicos y de negocios del proyecto.

En la mayor parte del desarrollo de los parques e incubadoras, se ha involucrado una región con sus autoridades y la universidad local, particularmente en la provisión de terrenos, infraestructura y la promoción misma de los desarrollos; estas acciones han estado normalmente vinculadas a una estrategia promocional de una región o ciudad. Se reconoce que la imagen y reputación de la universidad y su ambiente verde tipo parque, con poca edificación y diseños apropiados de los espacios físicos, son los elementos claves que permiten atraer a las empresas de alta tecnología.

Cabe indicar que, en los países industrializados, un número reducido de universidades, por si solas, se ha involucrado en parques. En la mayor parte de ellos, su puesta en marcha ha dependido de las agencias de desarrollo regional y autoridades locales pero sin el apoyo financiero, por lo cual estos planes han fracasado.

El sector privado, a su vez, ha orientado sus aportes de capital principalmente a través de las empresas en el sector de alta tecnología, antes que en el parque mismo. Sólo en pocos casos el sector privado lo ha hecho como propietario y arrendatario o como en el caso de algunos de ellos, por razones sociales de promoción u otras. Sin embargo, un número importante de parques en estos países está dirigido por el sector privado.

\section{Organización y formas de cooperación}

La razón básica que lleva a la universidad a organizarse para la cooperación con el medio en general, es poder articular, eficientemente, las demandas inmediatas o futuras del sector externo con las capacidades $u$ ofertas institucionales. Los esquemas de articulación no son únicos, dependerán del nivel de desarrollo y experiencia de la universidad como también, de la magnitud de los recursos y responsabilidades involucradas en la cooperación.

Por tanto, la universidad debe conocer las necesidades y demandas del sector productivo de bienes y servicios, para generar carteras de proyectos a fin de satisfacerlas y así paralelamente, esta institución académica pueda concretar acciones específicas de cooperación que produzcan beneficios económicos y académicos. Esto es posible de alcanzar en la medida en que la universidad desarrolle un proceso de gestión profesionalizado con una organización dinámica, en cuanto a las respuestas que del proceso se espera y un recurso humano comprometido con la causa.

La experiencia señala, claramente, que las empresas saben que pueden beneficiarse de los recursos y de la experiencia de la universidad, pero muchas veces la promoción o "marketing" de la oferta es deficiente, con esto la empresa tiene una visión muy parcializada de aquello que la institución educativa puede ofrecer. En este aspecto, el mutuo conocimiento es extrema- 
damente importante y éste puede lograrse mediante contactos regulares entre académicos y profesionales en proyectos conjuntos, proyectos de titulación, reuniones técnicas y programas de intercambio profesional. Esto lleva incluso a identificar falencias formativas en los futuros profesionales y a conocer las demandas reales por parte de la empesa, de programas de educación continua y de posgrado, servicios científicos y tecnológicos e I \& D.

Esta cooperación descansa fuertemente en la necesidad de la universidad en generar ingresos y acceder al financiamiento de programas de I\&D sobre la base de resultados, junto a la necesidad de la empresa de nuevos desarrollos tecnológicos para enfrentar la creciente competitividad y las demandas de los clientes.

Es necesario que la gestión se evalúe periódicamente, tanto desde el punto de satisfacción de los clientes internos (academia) y externos (empresas) para mantener claridad en su acción y una credibilidad a toda prueba ante los usuarios. Es decir, debe adquirir una marcada y reconocida orientación de "servicio para otros", por cuanto que su actividad corresponde a la de un canal de comunicación.

Es bueno que exista una sola "ventanilla institucional", pero a veces la confianza ha sido desarrollada directamente por el académico quien puede recurrir a su contacto directo, antes que a la "burocracia interna".

Las diferentes formas de cooperación entre la empresa, públicas o privadas, y la universidad pueden agruparse en cinco categorías principales:

\section{Consultoría académica}

Esta forma de cooperación se caracteriza por su clara orientación a resolver problemas de las empresas. La universidad desarrolla para ella actividades de consultoría y/o asistencia técnica, a través del trabajo de sus académicos más calificados para aplicar sus conocimientos en estudios específicos encomendados por la empresa.

Varios problemas técnicos del sector industrial y comercial necesitan de la opinión (informes técnicos) y apoyo del personal universitario experto en un área determinada (asesoría científica). Aparte de la capacidad académica, los precios bajos cobrados por la universidad comparados con los costos de las empresas de consultoría, si existen, constituyen una inne- gable ventaja para la misma institución educativa. Esta forma de cooperación es muy importante en la consolidación de vínculos universidad - empresa.

\section{Proyectos}

Dentro de esta categoría se encuentran:

Proyectos de investigación contratada: En este caso cuando una determinada empresa contrata con la universidad una investigación específica, en un campo donde ella es particularmente competente, el propósito de la investigación es tratar de crear un nuevo saber a partir de los conocimientos disponibles en la universidad. Esta cooperación es posible por cuanto que la empresa no mantiene actividades de investigación, a menos que sea imprescindible, como aquella dirigida al desarrollo de nuevos productos o servicios. Entonces, aparece la universidad con excelentes oportunidades y ventajas para desarrollar líneas de investigación financiadas por las empresas en áreas de las nuevas tecnologías. La universidad dispone, por lo general, de alta capacidad humana y de equipos, menores costos de administración y adecuado manejo de grandes proyectos.

En el mismo orden de cosas, algunos temas de investigación se adelantan por consorcios de empresas y universidades para licitar contratos específicos de investigación precompetitiva que conlleve al desarrollo de nuevos productos y procesos.

Otro tipo de investigación a realizar por la universidad con el patrocinio de empresas regionales, nacionales - multinacionales, podría dirigirse a trabajar en nuevas tecnologías de interés futuro (desarrollo de nuevos materiales, usos, etc).

Proyectos de innovación tecnológica: Se trata de innovaciones tecnológicas inducidas por la universidad en el mercado (la empresa puede recurrir a la universidad) o por interacción de los mismos. Aquí la transferencia de tecnología corresponde a una interacción entre la I \& D universitaria y la empresa, donde se transfieren competencias y propiedad intelectual desde la universidad. A través de estos proyectos se pueden adquirir nuevas capacidades que los investigadores pueden transferir hacia la universidad y al mismo tiempo, se generen nuevas líneas de I \& D y así, permitir a la academia trabajar más cerca del mercado. 
Esta tipología de proyectos, conocida como investigación aplicada, se caracteriza por ser un óptimo conducto de transferencia y difusión tecnológica.

\section{Programas de formaciōn de recursos humanos}

Dentro de esta categoría se observa, últimamente, un rápido aumento de los cursos relativos a la gestión de empresas en programas de maestría flexibles, ofrecidos por diversas universidades en horarios vespertinos. Ellos son de interés para las empresas pues pueden mejorar su capacidad administrativa, sin perder los servicios del personal durante largos períodos de la formación tradicional:

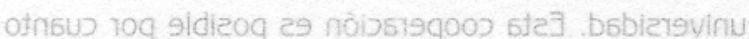
Las universidades, aparte de los programas antes señalados, ofrecen cursos cortos o de educación continua, especialmente concebidos para una empresa 0 un grupo de empresas. El costo de mantener una infraestructura de capacitación dentro de las empresas de países desarrollados lleva en muchas instancias a cuestionar si éste es el método más económico para llevarlos a cabo. Recurrir a un equipo externo para realizar cursos de formación en la propia empresa o en la universidad, es un medio más ahorrativo.

Debido al avance tecnológico actual, las empresas han incrementado su interés en cursos técnicos de posgrado y los de gestión. Sin embargo, en muchas ocasiones las universidades carecen de los enfoques prácticos, siéndo la preocupación de muchas empresas. Esto puede obviarse si la universidad crea centros de tecnología avanzada y unidades de capacitación de elevado nivel como por ejemplo: manufactura, diseño y manufactura asistida por computador (CAD/ CAM) y manufactura integrada por computador (CIM). Estos programas son una excelente forma de transferencia de tecnología, al tiempo que se mantiene al personal actualizado j̀ lonsst nöissvonni sb zojosyor bsbizysvinu st yog esbibubni essipòlonjet esnoibsvon

\section{Mecanismos institucionales}

En esta categoría se encuentran las siguientes modalidades:

Centros de investigación y unidades técnicas: La creación de estos mecanismos de transferencia y difusión tecnológica obedece al propósito de ofrecer soluciones rápidas a los problemas de las empresas, mediante la plena utilización de las capacidades humanas y de infraestructura. Ello no puede ofrecerse sobre esta base en los departamentos académicos de la

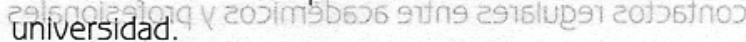



Las universidades pueden desarrollar estos centros para ofrecer servicios de $1 \& D$ cercanos al mercado de procesos y productos, cursos de actualización tecnológica, análisis, ingeniería de diseño de productos y procesos, nuevos materiales y robótica para mejorar la calidad y la competitividad de las empresas.

Empresas conjuntas: Esta modalidad de creaciôn puede ser de tipo más durable y de alta eficiencia. Muchas veces es posible identificar en la universidad proyectos de investigación cuyos resultados presentan ciertas perspectivas de ser explotados sobre una base comercial, donde la universidad no tiene fortalezas. En este caso, es posible una "joint-venture" entre la universidad y una empresa que trabaje en el rubro de probada experiencia en negocios y comercialización.


dad se asocie con una compañía deseosa de ejecutar un proyecto específico que requiere competencias que ella no posee, pero que logra identificar en una institución educativa. Así, académicos, jóvenes graduados debidamente seleccionados y profesionales de la empresa podrian trabajar por un tiempo en este tipo de proyectos de desarrollo a través de un acuerdo específico entre la universidad y una empresa.

Parques tecnológicos e incubadoras de empresas: La creación de estas formas de cooperación próximas a la universidad, con infraestructura de administración y espacio de alta calidad permitiría a las empresas, que se establezcan, beneficiarse de la presencia universitaria.

5xims̄b65 sinothano]

El interés de las empresás reside en ef acceso a las facilidades universitarias de alto nivel de calidad: intercambio de información, $P \& D$, utilización de ciertos laboratorios especializados, facilidades de bibliotecas, facilidades de espacio fisico y actividad en un medio estimulante y competitivo, entre otros beneficios.

Para los emprendedores o nuevos empresarios, las incubadoras, en general, les pueden facilitar el desarrollo de negocios emergentes, por la disponibilidad de servicios compartidos, el apoyo de la universidad en sus distintas formas y la disponibilidad de espacio físico y actividad en un medio estimulante y competitivo, entre otros. -9nni snu ngyutifenos, n9teixs ie, sinofluenos 9b z5a 
Para la propia universidad, estos mecanismos institucionales le ofrecen diversas alternativas como: atacar temas de investigación común, su promoción al medio, el desarrollo de recientes empresas y oportunidades de negocios para sus propios egresados o académicos y provisión de recursos financieros, entre otras.

\section{Otras formas de cooperación}

Licencias de explotación. Una forma tradicional de transferencia tecnológica ha sido la licencia de explotación de invenciones universitarias, a empresas en condiciones definidas contractualmente. La mayor parte de estas licencias provienen del campo de la ingeniería.

Servicios Científicos y Tecnológicos: Muchas veces estos se denominan simplemente servicios industriales y comprenden respaldo técnico en la solución de problemas de normalización y calibración, requerimientos de ensayos y pruebas industriales, selección de equipos, capacitación, diseño y desarrollo de maquinaria especializada y nuevos productos. Además de apoyar el uso de instrumental sofisticado.

Uso de instalaciones universitarias: Las universidades tienen una amplia gama de instalaciones y equipos de los cuales las empresas, normalmente, no disponen como: bibliotecas, salas de reuniones y equipos de investigación, entre otros. El uso de esta infraestructura puede permitir a las empresas beneficiarse en sus actividades de negocios y, a veces, en la recreación de su personal.

Proyectos específicos por financiación particular: Corresponde a proyectos generados, en ocasiones, por la existencia de instrumentos de financiación con carácter público que apoyan: 1) proyectos concertados entre la universidad y la empresa, 2) proyectos de empresas, 3) proyectos de universidades y centros tecnológicos, y 4) proyectos de investigadores de interés futuro para la empresa.

Reclutamiento de futuros profesionales: La realización de prácticas de verano de los alumnos, como complemento a su formación profesional, brinda una excelente oportunidad para que las empresas evalúen la posibilidad, en un futuro, de ofrecer empleo a estos estudiantes que tienen la posibilidad de mostrar sus habilidades mediante la realización de un buen trabajo, en un ambiente industrial o comercial. Este método ha resultado una excelente forma de reclutamiento de personal en lugar de la forma tradicional de ofrecer trabajo a través de los avisos de prensa. En numerosas ocasiones el estudiante en práctica puede inciar su trabajo de titulación en un tema aplicado, del interés de la industria, con lo cual son mayores los beneficios.

Por lo anterior, para articular y dinamizar la cooperación universidad-empresa y desarrollar actividades remuneradas con el sector externo, es necesario que la universidad estudie detalladamente aspectos relativos a: 1) El diseño de políticas orientadas a asumir la responsabilidad de prestar diversos tipos de servicios a la empresa, en áreas específicas, 2) La valoración de los servicios a la empresa en la carrera académica, 3) La adopción de formas específicas de contratos o convenios para la prestación, 4) El diseño de mecanismos para su administración, promoción y difusión, 5) Las decisiones en torno a temas de confidencialidad y propiedad del conocimiento generado, 6) La distribución y uso de los recursos generados por las diversas prestaciones, 7) Las responsabilidades institucionales para el manejo de la cooperción, y 8) El control de calidad y seguimiento de las actividades.

\section{Aspectos jurídicos de la cooperación}

Uno de los elementos asociados a la cooperación entre la universidad y la empresa, es el relativo a la claridad y formalización de los acuerdos entre la partes. Por ello, es necesario estudiar las formas y sus implicaciones para asegurar a los equipos de trabajo el respaldo institucional y proporcionar a la universidad una adecuada base de negociación. Para esto, es indispensable disponer de expertizaje jurídico dentro de la propia universidad para determinar las formas precisas que satisfagan a las partes contratantes.

La universidad tendrá interés en precisar que los compromisos que ella contrata no involucra a toda la institución, para evitar, de este modo, ciertos aspectos contractuales que se apliquen al conjunto de los investigadores universitarios.

\section{Objeto del contrato}

Es preciso que el objeto del contrato sea establecido con absoluta claridad por cuanto algunas responsabilidades comprometidas estarán influenciadas por su definición. Aquí no deberá confundirse el objeto del contrato con el campo cubierto por la investigación prevista. 


\section{Duración}

Por razones diferentes, cada una de las partes puede tener interés en suscribir convenios de larga duración. Para la empresa puede ser conveniente asegurarse una cooperación a largo plazo con la universidad para evitar que las inversiones que efectúe no se transformen muy pronto "de dominio público". Para la universidad un contrato de largo plazo permite asegurar la participación de sus investigadores y disponer de investigación estable en sus laboratorios. Sin embargo, existe el riesgo de que la universidad se convierta demasiado dependiente, en un campo específico, de una determinada empresa.

\section{Precio}

La universidad contrata con el propósito claro de autofinanciar total o parcialmente sus actividades de cooperación con la empresa para remunerar a sus académicos y financiar los gastos de sus laboratorios y crecimiento futuro. En la actualidad, pese a que las universidades no persiguen lucros, los precios establecidos en los contratos para realizar una investigación cubren los costos reales de ésta, incluyendo los gastos administrativos, financieros y de over-head.

\section{Obligaciones de la universidad:}

La universidad contrae tradicionalmente tres tipos de obligaciones: 1) La comunicación a la empresa sobre los resultados obtenidos; 2) La exclusividad, que es una obligación más delicada puesto que la empresa desea, justamente, tener la exclusividad sobre trabajos realizados por la universidad y, a su vez, la universidad desea también, legítimamente, guardar un máximo de independencia y libertad académica; 3) La obligación de confidencialidad, plantea algunos problemas delicados por cuanto la empresa desea conservar, por un período prolongado, los resultados en carácter confidencial mientras que la universidad desea hacer difusión de los mismos.

\section{Obligaciones de la empresa:}

Las obligaciones de la empresa son principalmente: cancelar los montos establecidos en los contratos dentro de los plazos, y aportar su concurso y colaboración a la realización de los trabajos.

\section{Propiedad de los resultados}

Una cooperación efectiva entre cada universidad y la empresa, involucra aspectos relativos a patentes y li- cencias; por ello, los arreglos deben comprender de alguna forma a personas (inventores) e instituciones participantes. El problema de la propiedad de los resultados es evidentemente muy importante y no existe una respuesta única. Casi siempre, dependerá de los aportes de las partes pero este argumento no es suficiente. La universidad no debe olvidar que sus académicos son indispensables en la generación de los resultados y ellos deben ser co-partícipes. En general, al suscribir el contrato de cooperación entre la universidad y la empresa, se habla de propiedad a partes iguales; este parece ser el criterio más aceptado para una satisfacción mutua. Distinta es la situación, cuando después de un trabajo contratado se produce una creación intelectual que excede el límite de lo esperado por la empesa en el marco del convenio suscrito; aquí la universidad y sus académicos pueden demandar todo el derecho.

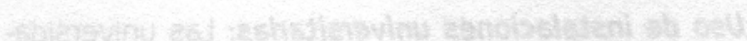

